\title{
RAPID PROTOTYPING OF MIXED REALITY APPLICATIONS THAT ENTERTAIN AND INFORM
}

\author{
Christian Geiger ${ }^{1}$, Christian Reimann ${ }^{2}$, Waldemar Rosenbach ${ }^{2}$, Jörg Stöcklein ${ }^{2}$ \\ ${ }^{1}$ Hochschule Harz, Wernigerode, Germany, cgeiger@hs-harz.de \\ ${ }^{2} C$-LAB, Paderborn, Germany,\{reimann,bobka,ozone\}@c-lab.de
}

\begin{abstract}
This paper describes a prototyping environment for rapid application development. We combine existing AR-technologies with a component-based 3D animation library and a scripting API. Through the development of an interface to a high-level 3D modelling system we are able to use this visual tool for modelling and basic animation features in MR design. This provides content experts with a powerful tool to quickly design and test mixed reality prototypes. We consider applications in the area of interactive mixed reality illustrations in the context of technical descriptions / user manuals and interactive exhibitions in museums.
\end{abstract}

Key words: Prototyping, Mixed Reality Design, 3D-Animation

\section{Introduction}

While technical advances in mixed reality are steadily increasing and solutions like ARToolkit [1] are available to interested researchers, there is still a lack of development tools for mixed reality applications. This unavailability requires content experts/authors to possess a broad range of technical programming skills and reduce the number of possible MR developers. Most often the development of new MR applications requires an ad-hoc implementation that is realized from scratch contradicting reusability and cost-efficiency and diverting the author's creativity from content to the underlying technology. Due to missing structured design approaches a tryand-error design is currently the best choice when developing mixed realities. Thus, rapid prototyping is very useful because authors can quickly

The original version of this chapter was revised: The copyright line was incorrect. This has been corrected. The Erratum to this chapter is available at DOI: 10.1007/978-0-387-35660-0_65 
develop alpha-versions of their applications and test and modify them without much overhead.

We identified the following requirements for efficient mixed reality prototyping.

- High-level access to computer generated media assets, i.e, the generation of 3D models and animations should be simplified

- Easy use of other media assets (images, sound, text)

- Reuse of computer generated content elements (e.g. 3D models)

- Scripting instead of programming provides short turn-around cycles

- Encapsulation of third-party AR-functionality and an efficient integration in the prototyping environment

In our projects we addressed these requirements and developed a prototyping environment based on our i4D system [3]. This allows i4D users to design AR-applications using the highlevel animation concepts of $14 \mathrm{D}$ together with its Tcl/Tk scripting API. Additionally, we developed an interface to the Maya animation package [11] that enables users to develop 3D models and simple keyframe animations with Maya and automatically import them in an interactive real time application.

\section{State of the Art in MR Authoring}

An early publication that considered the need for Augmented Reality Authoring was [6], where the generating and deploying of AR media is mentioned with regards to ARML, a data format that can supply the needs of a variety of AR displays or tool implementations. Studierstube is a 3D graphics library that has also been extended towards augmented reality [4].

ImageTcl [8] is a Multimedia Software Development Environment under development at Media and Entertainment Technology Laboratory at Michgan State University. ImageTclAR extends ImageTcl with new features in support of augmented and virtual reality development. It shares the idea of using a powerful scripting language, however it does not feature a high level library similar to i4D. Balicisoy et al presented a framework for the prototyping and evaluation of technical prototypes with augmented reality [7]. This approach focuses on the interaction of virtual humans with technical devices by extending 3D CAD models by additional object behavior that can be evaluated in an AR simulation running on SGI workstations. An authoring approach for the AR toolkit was presented in [9] as Tiles, a MR authoring interface for easy and effective spatial composition, layout and arrangement of digital objects in MR 
environments. An editor for marker-based AR software is also under development in the german ARVIKA project [5].

\section{Basic Concepts}

Our prototyping environment is based on three different concepts:

- A conceptual model that easily allows to design interactive mixed reality scenarios.

- A one-to-one mapping of the conceptual model to a high-level component-based library for MR applications that easily integrated thirdparty solutions.

- A script wrapper that allows to access the library's functions using $\mathrm{Tcl} / \mathrm{Tk}$

A conceptual model that fits dynamic and proactive content is the first component of our design approach. Conceptual models are used by designers both to reason about a design problem and to communicate with other stake holders (e.g. end-users), therefore they should be familiar to both groups. Since many conceptual models in multimedia design (cards, pages, books) do not match the dynamic and three dimensional nature of AR we have chosen an actor based model that was found to be very effective for our purposes. It is loosely based on the computers as theatre model by Laurel [2] and provides a common ground of techniques, vocabulary and metaphors from the domains of film and theatre. Virtual and real objects in the AR application are represented by actors. In contrast to virtual environment systems where all $3 \mathrm{D}$ objects are completely under the control of the designer the special characteristics of AR systems impose specific constraints on some objects and thus on the corresponding actors and their design. Therefore, we distinguish three classes of actors in our approach:

$R($ eal): R-actors correspond to real objects encountered in the environment in the final implementation of the AR application. R-actors serve mostly as triggers and inputs to the AR system. Their geometry and behaviour is not under the control of the designer. During prototyping and conceptual testing it is often necessary to introduce virtual place holders of $\mathrm{R}$ actors as $3 \mathrm{D}$ models with behaviour into the system to enable tests without the completely implemented system. Virtual R-actors could be provided as library components and reused across different concepts and applications.

$V($ irtual): $\mathrm{V}$-actors represent depictions of real-world objects that are virtually added to the real-world in the AR application, e.g. virtual batteries displayed over a real-world device in a maintenance application. They must be identifiable with their real-world counterpart and their behaviour should 
be sufficiently realistic to ensure comprehension on the user's side, but the exact geometry and behaviour is under the control of the designer.

$M$ (eta): $\mathrm{M}$-actors implement visualization techniques and artificial objects. They provide the visual and other multimedia information that is added to the real environment and can take any conceivable form which poses conceptual as well as geometric and behavioural design questions. Since $\mathrm{M}$-actors have no real-world reference and are free from constraints end-user participation and frequent verification of design assumptions through tests is essential. M-actors are not necessarily geometric, but can also perform abstract tasks, e.g. control of the graphics rendering system or coordination of other actors.

\section{Mapping Concepts to Tools}

A one-to-one mapping of the conceptual model to an efficient object oriented implementation is provided using our i4D system [3]. i4D is a highlevel component based library for interactive 3D animations. Following the actor based metaphor an i4D application consists of a number of actors placed on stages. Actors include conceptual objects like lights and cameras, visual 3D objects and software elements without a visual representation. Actors perform actions (e.g. animations, sending messages, sound) by continuously modifying their attributes. Conceptually a stage is viewed by a number of cameras and displayed on monitors which themselves are actor objects. The assignment of monitors, cameras, stages and actors can be altered interactively during runtime. Similar to scene graphs, actors can be hierarchically structured. In contrast to existing scene graph APIs, i4D provides access to high-level components that can be easily loaded into the system at runtime and can be combined to more complex building blocks. I4D allows to integrate third-party solutions. We easily integrated a number of applications as special i4D-actors, e.g. object loaders, video, a physical simulation engine or AR software. The latter allows to combine AR functionality of the ARtoolkit with the prototyping functionality of i4D.

Many 3D API's offer an efficient but less intuitive C++ API. For the design of interactive virtual worlds we found the technique of rapid prototyping very useful. To support this, a script binding (e.g. for Tcl/Tk, Python, Perl) allows to interactively design the virtual environment without compiling. We developed a scripting interface that allows to use i4D with arbitrary scripting languages. So far, we used Tcl/Tk and completely integrated the $\mathrm{i} 4 \mathrm{D}$ prototype in Tcl/Tk. Based on this work the development of visual tools for the design of i4D worlds is also very efficient. 
We developed a visual editor based on Tcl/Tk for i4D called "i4DDirector". This tools enables the user to place interactively actors on stages and modify their attributes. The scene editor allows a visual development of all i4D elements by providing special subeditor forms for each component type and combining them in configuration form. Due to the dynamic component loader in i4D it is also possible to load subeditors for specific components at runtime.

Furthermore the editor is able to handle unknown components which do not have an own subeditor for themselves at least at the scripting level. For our ARToolkit components we developed a special subeditor to simplify the alignment of virtual and real actors. Many designers are used to create their geometry and animations with tools like Alias Wavefront Maya. Thus, we developed an exporter for Maya to i4D which exports the modeled geometry and animations into an i4D-file. For geometry the Obj-file format is used and animation keys needed for interpolation are transformed into attributes for an $\mathrm{i} 4 \mathrm{D}$ action. At runtime the $\mathrm{i} 4 \mathrm{D}$ action interpolates the keys and executes the animation.
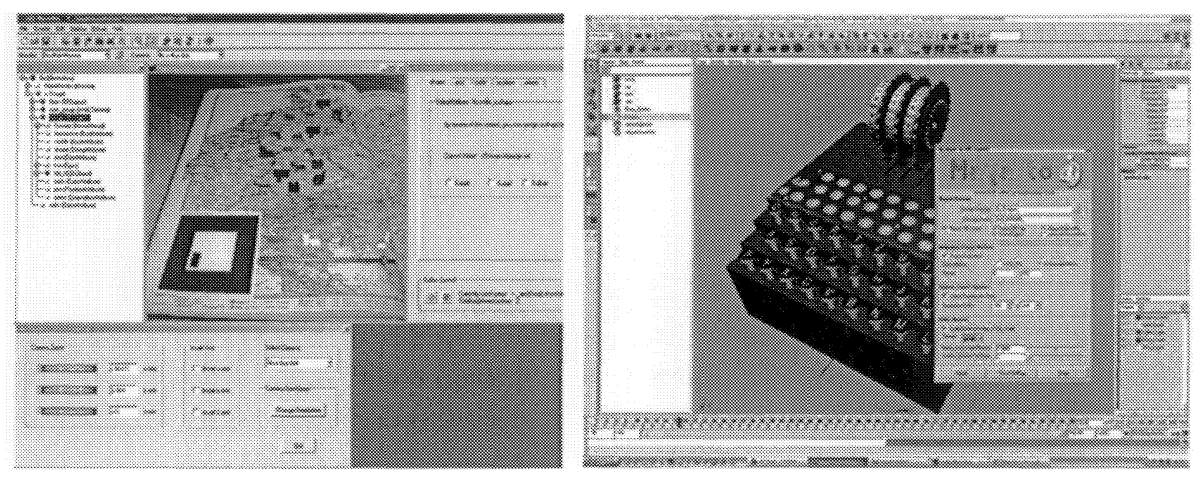

Figure 1: Tools for MR-Prototving

\section{Examples}

As a very simple example a Tcl script is presented that creates a "HelloARWorld": if the specified pattern is recognized a simple red cube is projected.

load Illu // load i4D library into Tcl

Create Stage $s$

$s$ Create Camera c

s Create LiveCam Ic

MainMonitor SwitchTo s.c

s.background s.lc

s Create DirectionalLight dl orient (( $\left.\begin{array}{lll}1 & 1 & 1\end{array}\right)$ 30) s Create RealObject ro1

s.ro1 Create 3DObject Cube o1 color ( $\left.\begin{array}{lll}1 & 0 & 0\end{array}\right)$

$s$ Create PatternRecognition pr

s.pr InsertPattern s.ro2 "Datallpatt.sample1"

s.pr.picture s.lc

s.Ic Start 
We developed a number of more complex examples using our current prototyping environment. They were mainly driven by "demo requirements" and thus had to be developed in very short time. The DigiCam example
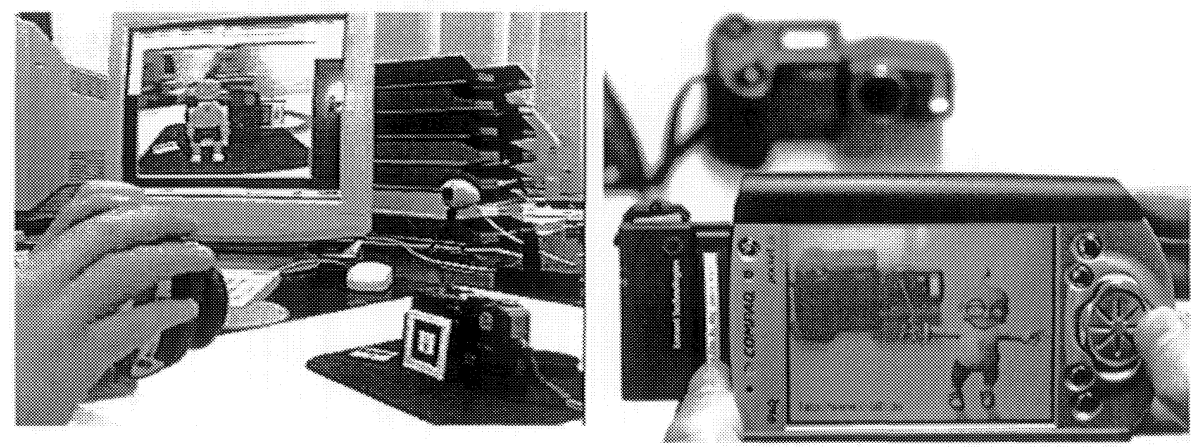

Figure 2: AR-Illustration "DigiCam"

features an animated character that explains how to insert a CFII-card into a digital camera. By using a special R-actor bbcamera, that serves as invisible bounding box for the camera it was possible to occlude virtual objects by real ones (e.g. by rendering the bbcamera actor into the Z-buffer but not into the frame buffer). The application was rendered on a PC running i4D on Linux. Using i4D's off-screen render facilities (hardware accelerated using nvidia P-buffer) frames were sent via a wireless LAN to a mobile client that renders them. Animations and models were first developed using the Maya animation tool and then imported into the i4D prototyping environment. Another more complex infotainment example features an interactive illustration for a museum artefact at the world's largest computer museum in Paderborn, Germany.

The well know encryption machine ENIGMA, used during world war II is augmented by an information tour that explains the complex crypting mechanism. This uses virtual, real and meta actors (e.g. virtual cables and pins for interactive coding messages in virtuality) developed in i4D to
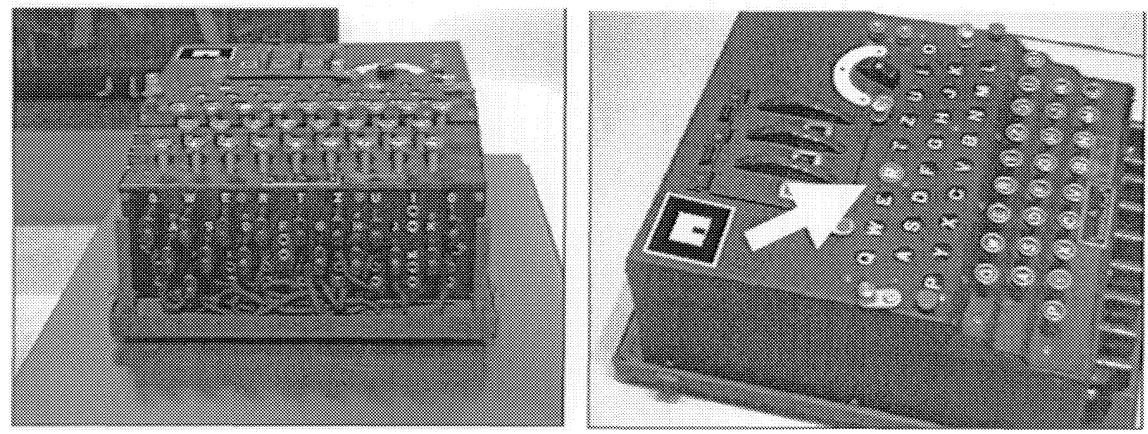

Figure 3: AR-Enigma encrypting letter "R" (arrow inserted for this paper) 
interactively present the features of this historic machine. The ARillustration highlights buttons that are virtually pressed by the user and shows the encryption process by using virtual 3D-copies (ghost objects) of some enigma parts to animate this.

\section{Conclusion and Future Work}

This paper addressed the design of mixed reality applications from a "prototyping" point of view. With the described approach we want to support content experts that are not experienced programmers of MR applications. The combination of commercially available modeling and animation tools with a custom high level 3D animation library that featuring a scripting API is a first step in this direction as current examples showed. Although not described here, our work also includes a user centred design process that closely follows the described actor metaphor and that considers iterative prototyping. For the future we will continue to extend this approach with usability engineering techniques that allow for intensive user testing [10]. Following the idea of a prototype's "testable design representation" at each step in the design we work on tool for non real-time mixed reality representations for our system. During design phases actors may be represented as text, sound, augmented images, video or animated 3D objects. The designer should be able to switch between different actor representations based on his current focus and refinement level.

\section{Acknowledgements}

Volker Paelke, Dirk Stichling and Bernd Kleinjohann have provided valuable input to results described in this paper.

\section{References}

[1] ARToolkit Download Page: http://hitl.wahington.edu/research/shared_space

[2] B. Laurel, Computers as Theatre, Addison Wesley, NewYork, 1991

[3] Geiger, C., Paelke V., Reimann, C., Rosenbach, W.: A Framework for the Structured Design of VR/AR Content, "Proc. of the ACM Symposium on Virtual Reality Software \& Technology, VRST 2000", Seoul, Korea, 2000

[4] Studierstube Home Page. http://www.studierstube.org

[5] Wolfgang Friedrich, ARVIKA Augmented Reality for Development, Production, and Service, Tagungsband des Informationsforum Virtuelle Produktentstehung (IVIP), May, 2000.

[6] Ulrich Neumann and Anthony Majoros. "Cognitive, Performance, and Systems Issues for Augmented Reality Applications in Manufacturing and Maintenance." Proceedings of IEEE VRAIS'98, pp. 4-11, 1998. 
[7] Selim Balcisoy, Marcelo Kallmann, Pascal Fua, Daniel Thalmann. A Framework for Rapid Evaluation of Prototypes with Augmented Realit, The Symposium on Virtual Reality Software \& Technology 2000, Seoul, Korea

[8] ImageTcl Home Page: http://metlab.cse.msu.edu/imagetcl/

[9] Poupyrev, I., Tan, D.S., Billinghurst, M., Kato, H., Regenbrecht, H., Tetsutani, N. (2001). Tiles: A Mixed Reality Authoring Interface. INTERACT 2001 Conference on Human Computer Interaction, Tokyo, Japan.

[10] C. Geiger, V. Paelke, C. Reimann, J. Stöcklein. User Centred Design Space Exploration for Consumer AR Applications, OzCHI 2001, November 2001, Fremantle, Australia

[11] Alias Wavefront. http://www.aliaswavefront.com 